\begin{tabular}{|c|l|}
\hline Title & Topology optimization of wideband array antenna for microwave energy harvester \\
\hline Author(s) & Mori, Takuya; Igarashi, Hajime \\
\hline Citation & $\begin{array}{l}\text { International journal of applied electromagnetics and mechanics, 52(1-2), 631-639 } \\
\text { https://doi.org/L0.3233/JA_E-162057 }\end{array}$ \\
\hline Issue Date & 2016 \\
\hline Doc URL & http://hdl.handle.net/2115/64700 \\
\hline Rights & The final publication is available at IOS Press through http://dx.doi.org/10.3233/AAE-162057 \\
\hline Type & article (author version) \\
\hline File Information & ISEM2015_Full_tmori_ver4.pdf \\
\hline
\end{tabular}

Instructions for use 


\title{
Topology Optimization of Wideband Array Antenna for Microwave Energy Harvester
}

\author{
Takuya Mori and Hajime Igarashi \\ Graduate School of Information Science and Technology, Hokkaido University, 060-0814, Japan
}

\begin{abstract}
This paper presents optimal shape design of planar array antennas for microwave energy harvesters. The topology optimization of the $C_{4}$-symmetric array antenna is conducted to maximize their isotropic gain and minimize the return losses in a frequency band ranging from 1.5 to $3.5 \mathrm{GHz}$. Then, we design the rectifier by varying the circuit parameter based on measurement results to realize the impedance matching between the optimized antenna and rectifier. It is shown by experiment that the harvester composed of the optimized array antenna and rectifier has higher receiving voltage in comparison with the single one. Moreover, the resultant harvester is found to start operating when the arrival electric field is greater than $0.1 \mathrm{~V} / \mathrm{m}$.
\end{abstract}

Keywords: Microwave energy harvester, wideband antenna, topology optimization, array antenna

\section{Introduction}

In recent years, wireless sensors have attained great attentions for monitoring health and safety conditions of infrastructures such as bridge, tunnel and rail ways. When wireless sensors have batteries as their power supply, it is laborious and expensive to replace huge number of the batteries in the sensors distributed in environment. Therefore, wireless sensors which can operate autonomously are required.

The microwave energy harvesting devices which absorb energy from ambient microwaves are promising for the energy sources of the wireless sensors [1-3]. The microwave energy harvester is composed of a receiving antenna and rectifier. Arrival microwave is received by the antenna and the rectifier converts the received microwaves into DC power. To develop the high-efficiency microwave energy harvester, the antenna shape and circuit parameters are optimally designed.

For efficient power generation, the authors have developed the spiral antenna as well as $C_{4}$-symmetric antenna whose shapes are determined by parameter and topology optimizations, respectively [4]. It has been found that the $C_{4}$-symmetric antenna has better characteristics. We expect that the performance would be further improved by arraying $C_{4}$-symmetric antennas.

In this study, we develop a microwave harvester which includes an array antenna composed of four identical $C_{4}$-symmetric antennas. The shape of the $C_{4}$-symmetric antenna is determined by topology optimization using the micro genetic algorithm ( $\mu \mathrm{GA})[5]$ to realize high-gain and wideband characteristics. The performance of the antenna is computed by FDTD method [6]. For comparison, a single $C_{4}$-symmetric antenna is also developed by topology optimization. We here focus on energy harvesting from microwaves generated by a wireless rooter using the optimized antenna, whose frequency is assumed to be $2.45 \mathrm{GHz}$. We determine the size of the rectifier and stub length so that it is matched to the antenna at $2.45 \mathrm{GHz}$ by varying the circuit parameter based on measurement results. We test the performance of the developed microwave harvester by experiments.

\section{Antenna analysis for array antenna}

The frequency characteristics of antennas are computed using FDTD method [6] in this study, which allows us to effectively compute the frequency characteristics in a wide band. Let us consider the Maxwell equations

$$
\nabla \times \boldsymbol{E}+\mu \frac{\partial \boldsymbol{H}}{\partial \boldsymbol{t}}=0
$$




$$
\nabla \times \boldsymbol{H}-\varepsilon \frac{\partial \boldsymbol{E}}{\partial \boldsymbol{t}}=\boldsymbol{J}
$$

where $\boldsymbol{E}, \boldsymbol{H}, \boldsymbol{J}, \mu$ and $\varepsilon$ are electric field, magnetic field, current density, permeability and permittivity, respectively. Equation (1) is discretized with the FDTD method where time and space derivatives are approximated by central finite differences.

In this study, the antenna is printed on a dielectric substrate whose relative permittivity and thickness are set to 4.4 and $2.0 \mathrm{~mm}$, respectively. The Gaussian pulse is fed at the feeding port of the antenna and its input impedance is computed from the Fourier transform of the input voltage and current as follows:

$$
Z_{\text {in }}(f)=\frac{F\left[V_{\text {in }}(t)\right]}{F\left[I_{\text {in }}(t)\right]}
$$

where $V_{\text {in }}(t)$ and $I_{\text {in }}(t)$ are the feeding voltage and current obtained by the FDTD analysis. For analysis of the array antenna, Gaussian pulses are fed simultaneously at the feeding points of each antenna to consider the interference each other. The other conditions in FDTD analysis are summarized in Table I.

Table I

FDTD conditions in the optimization

\begin{tabular}{cc}
\hline \hline $\mathrm{NX} \times \mathrm{NY} \times \mathrm{NZ}$ & $150 \times 60 \times 150$ \\
\hline$(\Delta X, \Delta Y, \Delta Z)$ & $(1.0,1.0,1.0)[\mathrm{mm}]$ \\
\hline$\Delta t$ & $1.9 \times 10^{-12}[\mathrm{sec}]$ \\
\hline Number of time steps & 3000 \\
\hline Boundary condition & Perfect Matched Layer (10 layers) \\
\hline \hline
\end{tabular}

\section{Topology optimization method of wideband array antenna using NGnet}

For the topology optimization, we represent the antenna shape with a linear combination of the normalized Gaussian functions given by [8]

$$
\begin{aligned}
& y(\boldsymbol{x})=\sum_{i=1}^{N} w_{i} b_{i}(\boldsymbol{x}) \\
& b_{i}(\boldsymbol{x})=G_{i}(\boldsymbol{x}) / \sum_{k=1}^{N} G_{k}(\boldsymbol{x})
\end{aligned}
$$

where $G_{k}(\boldsymbol{x})$ and $\boldsymbol{w}_{i}$ denote the Gaussian function and weighting coefficient, respectively (see Fig. 1). To express the $C_{4}$-symmetric shapes, we introduce the unit region, symbolized by " $\boldsymbol{F}$ ", and the centers of 81 Gaussian functions are uniformly deployed so that the domain is covered by the support of the Gaussians as shown in Fig. 2(a). The antenna shape in the unit region is determined by optimizing the weighting coefficients $w_{i}$ in (3) using $\mu \mathrm{GA}$. The edge length of the FDTD cubic cell is set to $1 \mathrm{~mm}$. The antenna is modeled as a perfect conductor in the FDTD computation. The state $s_{e}$ of each FDTD-cell at its center $\boldsymbol{x}_{e}$ is determined from $y$ as follows:

$$
s_{e}= \begin{cases}\text { conductor } & y\left(\boldsymbol{x}_{e}\right) \geq 0 \\ \text { air } & y\left(\boldsymbol{x}_{e}\right)<0\end{cases}
$$

A $C_{4}$-symmetric antenna is modeled by introducing point symmetry around the feeding point surrounded by four unit regions as shown in Fig. 2(b) and (c). The array antenna is composed of four $C_{4}$-symmetric antennas. For comparison, the single $C_{4}$-symmetric antenna is also considered as shown in Fig.2(c). The standard deviations of $G_{i}$ are set to $24 / 16 \mathrm{~mm}$ and 50/16mm for the topology optimization of the array and single antenna, respectively. 

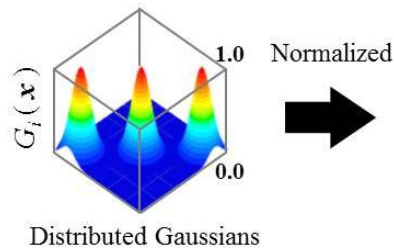

Distributed Gaussians

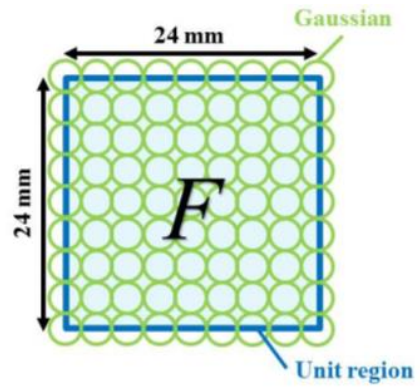

(a) Gaussian distribution in the unit region

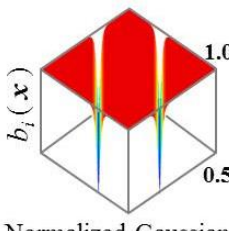

Normalized Gaussians

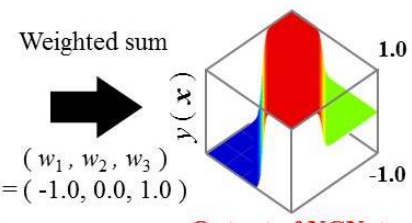

Output of NGNet

Fig. 1. The output of NGnet.

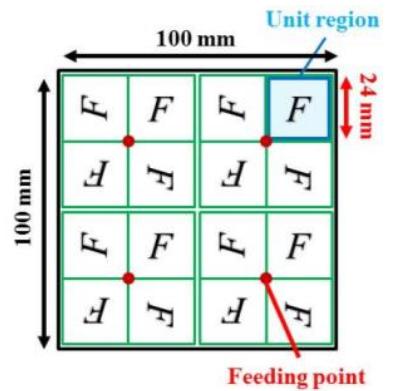

(b) Modeling of the $C_{4}$-symmetric array antenna

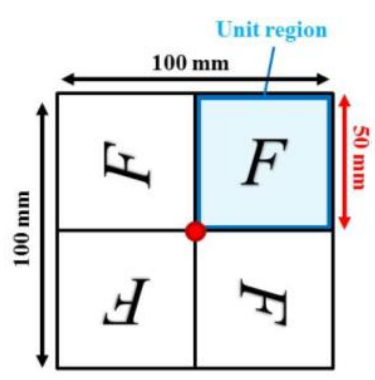

(c) Modeling of the single $C_{4}$ symmetric antenna

Fig. 2. Modeling for the $C_{4}$-symmetric antennas using NGnet.

\section{Optimization problem}

The antenna shape is optimized to maximize the isotropic gain $G_{\text {iso }}$ in consideration of mismatch losses between the antenna and circuit over the frequency band ranging from $f_{0}$ to $f_{1}$. For this purpose, we introduce the actual gain $G_{\text {actual }}$ to define the optimization problem as follows:

$$
\begin{gathered}
<G_{\text {actual }}>=\frac{\int_{f_{0}}^{f_{1}} G_{\text {actual }}(f) \mathrm{d} f}{f_{1}-f_{0}} \rightarrow \max \\
G_{\text {actual }}(f)=G_{\text {iso }}(f)\left(1-\left|\frac{Z_{\text {in }}(f)-Z_{0}}{Z_{\text {in }}(f)+Z_{0}}\right|^{2}\right)
\end{gathered}
$$

where $Z_{\text {in }}(f)$ is the input impedance of the antenna. In the optimization, $f_{0}, f_{1}$ and $Z_{0}$ are set to $1.5 \mathrm{GHz}$, $3.5 \mathrm{GHz}$ and $50 \Omega$, respectively. The gain and input impedance are computed by the FDTD method. We use $\mu \mathrm{GA}$ [5] to solve the optimization problem (6), in which the number of individuals is set to 8 and the optimization processes are continued for 100 generations. In this setting it takes about 4 days to obtain the final results using the Intel Xeon $C P U(2.4 \mathrm{GHz}, 8$ cores $)$.

\section{Optimization results for wideband antenna}

The resultant shapes of the optimization are shown in Fig. 3 and the return losses between the optimized antenna and $50 \Omega$ resistance are shown in Fig. 4 where the numbers from 1 to 4 represent the position of antennas in the array antenna. Their actual gains are also plotted against frequency in Fig. 5. It is indicated in Fig. 4 that the return losses of the array antenna are less than $-10 \mathrm{~dB}$ over the target frequency range while the single antenna has larger mismatch losses. It is found from Fig. 5 that the actual gain of the optimized $C_{4}$-symmetric array antenna is higher than that of the single antenna, and the former is more than $5 \mathrm{dBi}$ over the target frequency range.

We evaluate the power received by the array antenna illuminated by plane waves using FDTD analysis. In the analysis, the frequency of the plane wave and electric field intensity are set to $2.45 \mathrm{GHz}$ and $10 \mathrm{~V} / \mathrm{m}$, respectively. To consider the effect of the impedance matching between the antenna and circuit, the feeding port is loaded with resistance of $50 \Omega$. 
The voltages induced at the load resistances are shown in Fig. 6 where the received voltage of the array antenna is the linear sum of the voltages induced by four $C_{4}$-symmetric antennas. We find that the amplitude of received voltage of the array antenna is about 1.7 times higher than that of the single one. Accordingly, the received power is increased by about 3.0 times by using the array antenna.

\section{Measurement results}

\subsection{The frequency characteristics of the optimized antennas}

The computed and measured frequency characteristics of the input impedances $Z_{\text {in }}$ of the array and single antennas are plotted in Fig. 7. They are printed on the dielectric substrate made of FR-4 and we used E5061B Network Analyzer for measurement. From these figures, we find that there are some discrepancies between the computed and measured profiles. These discrepancies would mainly be attributed to the manufacturing error and unexpected losses of the dielectric substrate. Then, we design the rectifier considering impedance matching to the optimized antennas at $2.45 \mathrm{GHz}$ based on these measurement results.

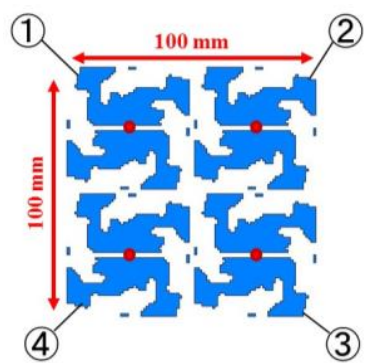

(a) Array
(3)

Fig. 3. Optimized shapes of each antenna.

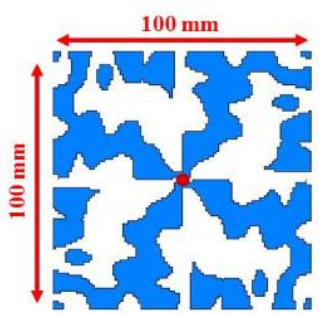

(b) Single

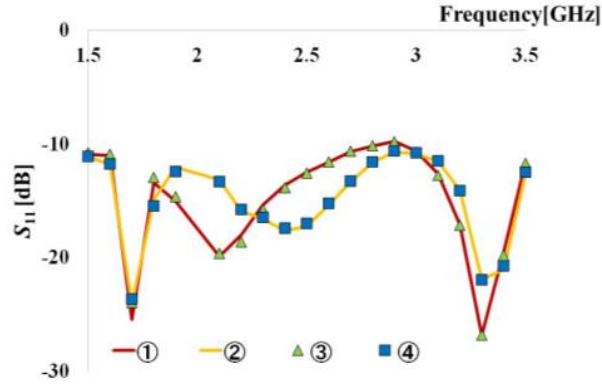

(a) Array

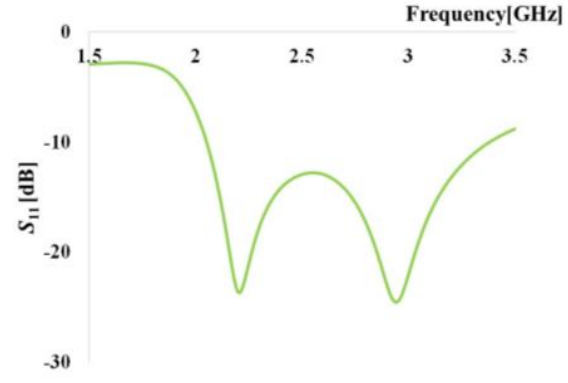

(b) Single

Fig. 4. Computed return losses of each antenna.

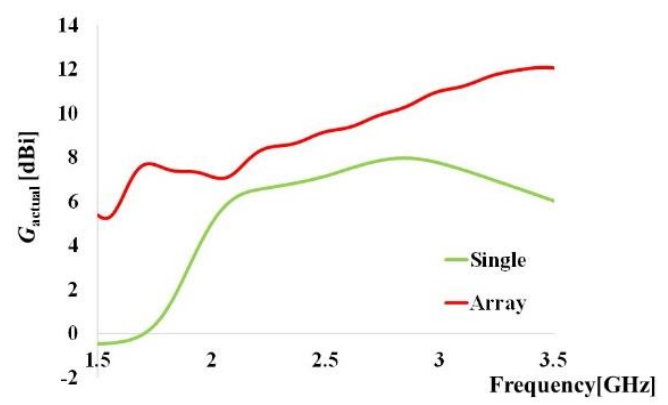

Fig. 5. Computed actual gains of each antenna.

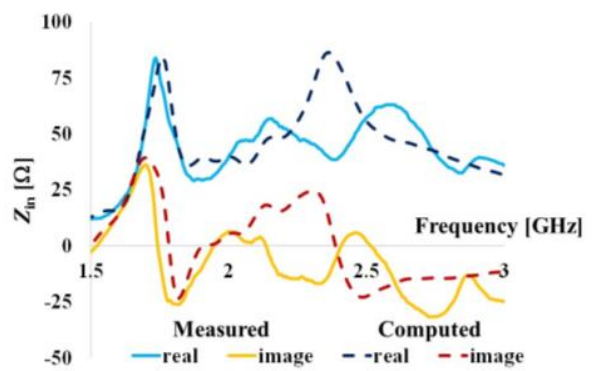

(a) Array

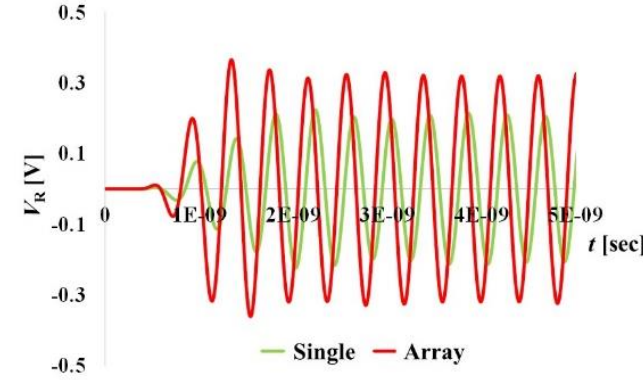

Fig. 6. Computed received voltages.

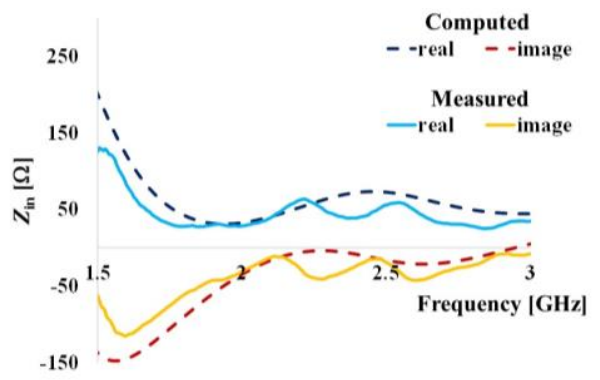

(b) Single

Fig. 7. Measured input impedance of each antenna 


\subsection{Design of rectifier}

For the rectifying circuit, we employ the single-stage full-wave Greinacher rectifier [9] shown in Fig. 8. For the diodes, we employ HSMS285x zero-bias Schottky-barrier diode [10] and the capacitance is set to 100pF. In this study, the open stabs are add to this rectifier (see Fig. 8(b)) and we determine the overall size of the rectifier and the stab length $l$ by trial and error to have impedance matching between the antenna and rectifier at $2.45 \mathrm{GHz}$. It is found that the array and single antenna have the minimum return losses when $l$ is $15 \mathrm{~mm}$ and $8 \mathrm{~mm}$, respectively. The measured return losses between the each antenna and designed rectifier are shown in Fig. 9. From the figure, both return losses at $2.45 \mathrm{GHz}$ are lower than $-20 \mathrm{~dB}$.

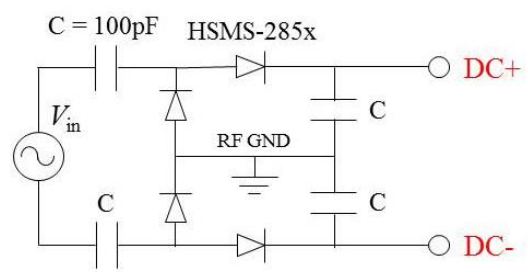

(a) Circuit diagram

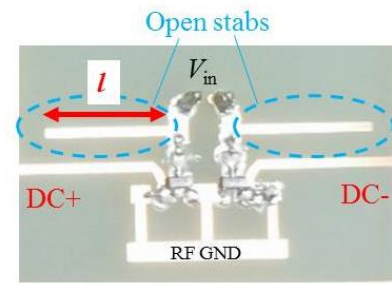

(b) Manufactured rectifier

Fig. 8. Design of the single-stage full-wave Greinacher rectifier.

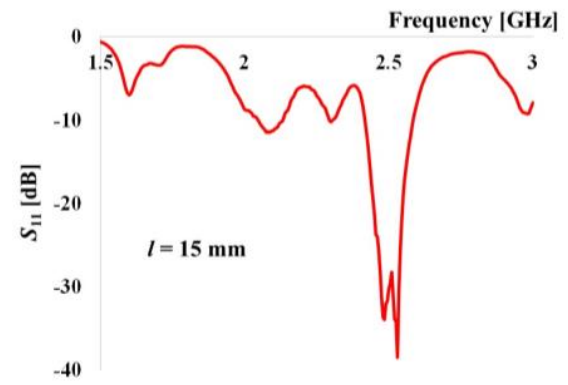

(a) Array

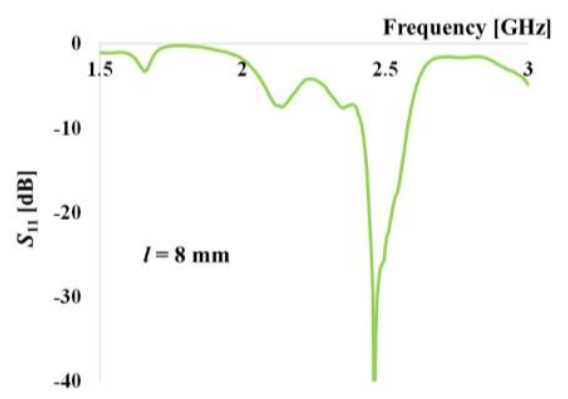

(b) Single

Fig. 9. Measured return loss $S_{11}$ between the antenna and circuit.

\subsection{Radio reception experiment}

The manufactured microwave harvester composed of the array antenna and the rectifier circuit printed on the back side is shown in Fig. 10. The harvester which contains the single antenna is also developed. They are printed on the same dielectric substrate (FR-4). The microwave radiated by the horn antenna is received by these harvesters as shown in Fig. 11. The distance between the harvesters and the transmitting horn antenna is set to $50 \mathrm{~cm}$ and the frequency of microwave is set to $2.45 \mathrm{GHz}$.

The measured output voltages of the rectifier is plotted versus the input electric field evaluated from Friis formula in Fig. 12. It is clearly seen from Fig. 12(a) that the output voltage of the harvester with the array antenna is higher than that with the single one. For example, when the electric field intensity $E$ is $10 \mathrm{~V} / \mathrm{m}$, the former output voltage is about 1.5 times higher than that of the latter. These results are consistent with the analysis results. From Fig. 12(b), it is found that when the electric field strength is larger than about $0.1 \mathrm{~V} / \mathrm{m}$, both harvesters can work. This suggests that these harvesters can get power from the microwaves radiated by usual rooters in indoor environment [7].

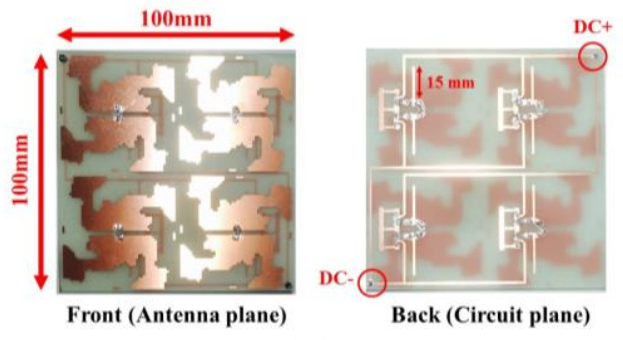

(a) Array

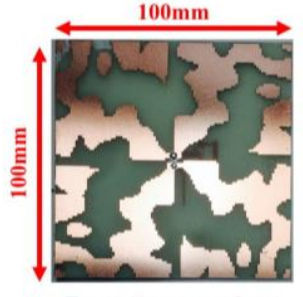

Front (Antenna plane)

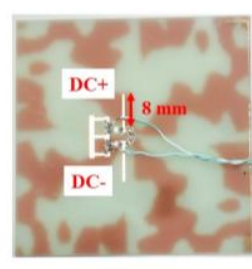

Back (Circuit plane)

(b) Single

Fig. 10. Manufactured harvesters composed of optimized antennas and rectifiers. 


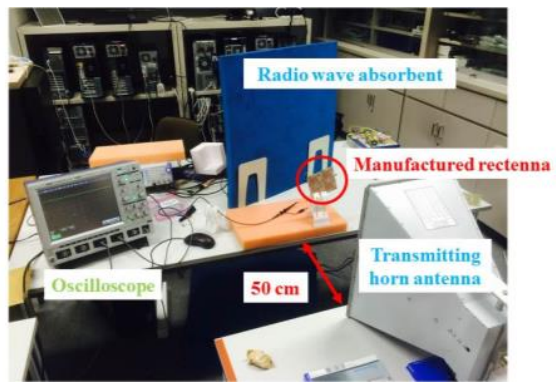

Fig. 11. Experimental setup.

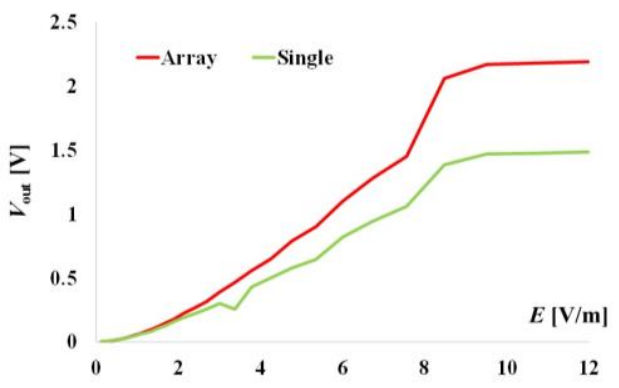

(a) over all

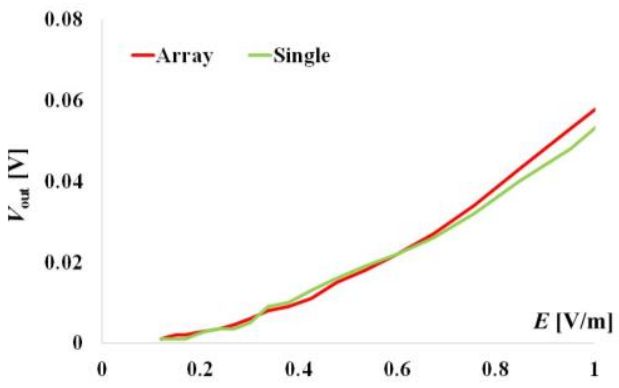

(b) enlarged view

Fig. 12. Measured output voltages $V_{\text {out }}$ of each harvester.

\section{Conclusions}

In this paper, we have presented optimal antenna design for microwave energy harvesters. The shapes of the array and single antenna have been determined so that the actual gain is maximized. Actual gains and return losses from $1.5 \mathrm{GHz}$ to $3.5 \mathrm{GHz}$ of optimized $C_{4}$-symmetry array antenna are more than $5 \mathrm{dBi}$ and less than $-10 \mathrm{~dB}$, respectively. The rectifier has been designed by trial and error based on measured frequency characteristics of the optimized antenna aiming at impedance matching. The measured receiving voltage of the harvester composed of the array antenna is higher than that of the single one by about 1.5 times. These harvesters start working when the input electric field is stronger than about $0.1 \mathrm{~V} / \mathrm{m}$. We will implement the power management system in these harvesters and use it as a power source of a wireless sensor.

\section{Acknowledgements}

This work was supported in part by JSPS KAKENHI Grant Number 15H02976, as well as JSPS and CAPES under the Japan-Brazil Research Cooperative Program.

\section{References}

[1] D. Bouchouicha, et. al,, "Ultra-efficient microwave harvesting system for battery-less micropower microcontroller platform," IET Microw. Antennas Propag., vol. 5, 1ss. 7, pp. 811-917, 2011.

[2] K. W. Lui, et. al,, "Ambient RF Energy Harvesting," International Conference on Renewable Energies and Power Quality (ICREPQ'10), March, 2010.

[3] R. Shigeta, et. al,, "Ambient RF Energy Harvesting Sensor Device With Capacitor-Lakage-Aware Duty Cycle Control," IEEE Sensors Journal, vol.13, no. 8, pp. 2973-2983, August, 2013.

[4] T. Mori, et. al, "Shape Optimization of Wideband Antennas for Microwave Energy Harvesters Using FDTD" IEEE Trans. Magn., vol. 51, No. 3, March, 2015.

[5] C. Coello and G. Pulido, "A Micro-Genetic Algorithm for Multiobjective Optimization," Lecture Notes in Computer Science, Volume 1993, pp. 126-140, 2001.

[6] A. Taflove. Computational Electrodynamics: The Finite Difference Time-Domain Method. Norwood, MA: Artech House, 1996.

[7] H. J. Visser, et al., “Ambient RF Energy Scavenging:GSM and WLAN Power Density Measurements", Proc. The $38^{\text {th }}$ European Microwave Conference, pp. 721-724, 2008.

[8] T. Sato, et. al, "Topology Optimization Considering Multimaterial Distributions Based on Normalized Gaussian Network," IEEE Trans. Magn., vol. 51, No. 3. March, 2015.

[9] U. Olgun, C. - C. Chen, J. L. Volakis, "Design of an efficient ambient WiFi energy harvesting system" IET Microwaves, Antennas \& Propagation, vol. 6, lss. 11, pp. 1200-1206, 2012.

[10] Avago Technologies, "HSMS-285x surface mount RF Schottky barrier diodes," http://www.avagotech.com/docs/AVO02-1377EN, 2010. 